\title{
Point-of-Care Research: Retrospective Analysis of the Evaluation and Classification of Tendon Pathology in Athletic Training
}

Ashley J. Reeves, DAT, AT*; Russell T. Baker, PhD, DAT, AT, CMP, PRT-c*; Scott W. Cheatham, PhD, DPT, PT, OCS, AT, CSCSt; and Alan Nasypany, EdD, AT*

*University of Idaho, Moocow, ID †California State University Dominguez Hills, Carson, CA

\begin{abstract}
Tendon pathology has been studied across healthcare professions but remains poorly understood. Imaging and clinical findings have been used to diagnose tendon pathology, but these findings are discrepant. It is vital that clinicians use sound clinical judgment to determine the most accurate clinical diagnosis and treatment options given documented clinical findings. The purposes of this study were to assess athletic trainers': 1) documented clinical findings for patients presenting with tendon pain, 2) use of documented findings to inform clinical diagnosis of tendon pathology, and 3 ) change in tendon pathology classification when presented with a novel diagnostic term. A total of 430 patients ( 20.70 $\pm 7.35 y$ ) from a multisite research database were included in the study. Pain at the site of injury was documented in $95.8 \%$ of cases $(n=412)$. Pain during exercise that changed activity ( $n=274,63.7 \%$ ), and an identified tender point ( $n$ $=259,60.2 \%$ ) were also present in almost two-thirds of cases. Of the patients diagnosed with tendinitis, $35.0 \%$ had pain as the only documented inflammatory sign. Of the initial set of clinical diagnosis options, tendinopathy was the most commonly $(n=290,67.4 \%)$ selected. There was a $46.0 \%$ and $15.0 \%$ decrease in the number of tendinopathy and tendinitis diagnoses, respectively, when 'tendinalgia' was an option as a diagnostic classification term. There does not appear to be adequate clinical evidence to label tendon pathology as either inflammatory or degenerative. Furthermore, clinicians either appear to be: 1) relying on few symptoms to identify a diagnosis or 2) not at first fully considering all clinical findings when diagnosing a patient. According to these findings, tendinalgia seems to be the most appropriate term to describe tendon pain, to help clinicians understand tendon pathology as a pain condition rather than as inflammatory or degenerative.
\end{abstract}

\section{Key Phrases}

Diagnostic testing, upper extremity, tendinopathy, clinical reasoning

\section{Correspondence}

Dr. Ashley Reeves, University of Idaho, 875 Perimeter Drive MS 2401

Moscow, ID 83844.

E-mail: reevesa@uidaho.edu

\section{Full Citation}

Reeves AJ, Baker RT, Cheatham SW, Naypany A. Point-ofcare research: Retrospective analysis of the evaluation and classification of tendon pathology in athletic training. Clin Pract Athl Train. 2020;3(2):33-44. https://doi.org/1031622/2020/0002.5.

Submitted: August 1, 2019 Accepted: March 2, 2020

\section{INTRODUCTION}

T endon pathology is a common problem among physically active individuals, but is poorly understood by researchers and clinicians alike. ${ }^{1-3}$ Previously, pain at a tendon has been termed tendinitis or tendinosis, describing either an inflammatory or degenerative condition, respectively. ${ }^{3-6}$ Tendinitis involves signs and symptoms of inflammation, which include heat, redness, pain, swelling, and decreased function. ${ }^{3-}$ 9 Clinicians grade tendinitis from first to third degree based on the severity and consistency of the patient's symptoms. ${ }^{10}$ Generally, symptoms become progressively more persistent as the condition worsens, advancing from pain noted only after activity to more consistent pain and decreased performance. ${ }^{10}$ Even though tendon pain is often referred to as tendinitis, tendinosis may be a more correct description, as evidenced by the lack of inflammatory markers in histological studies.3,6,11 In contrast to tendinitis, which describes a primarily inflammatory condition, tendinosis describes changes in tissue integrity (i.e., degeneration) without signs of active inflammation. $3-6,12,13$ Tendinosis is categorized according to the assumed severity of the condition, with Stage I correlating to transient pathology and Stage II correlating with more lasting changes to less than half of the tissue structure. ${ }^{14}$

Imaging studies (e.g., magnetic resonance imaging [MRI] and diagnostic ultrasound) provide further 
evidence of the potential inaccuracy of a tendinitis diagnosis, in which imaging findings are often uncorrelated to patient symptoms.3,15-23 For example, in a sample of 253 healthy, asymptomatic individuals ages 13-89 years old, $65 \%$ of individuals had tissue abnormalities of the proximal hamstring tendon on MRI $(13 \%$ unilateral, $52 \%$ bilateral). ${ }^{23}$ Of note, none of the participants had a history of hamstring pathology. ${ }^{23}$ In a second study, which involved ultrasound examination of 51 asymptomatic males, $96 \%$ had some form of pathological change to the rotator cuff. ${ }^{20}$ Of those individuals with positive findings, $75 \%$ had rotator cuff pathology, with the most commonly affected structure being the supraspinatus tendon $165 \%$ of total; 5 full thickness tears, 12 partial-thickness tears). ${ }^{20}$ These findings indicate that pathological changes to the tissue may not be the actual cause of a patient's symptoms, if asymptomatic individuals have the same tissue abnormalities on imaging studies that would typically only be expected in symptomatic patients. $3,15-23$

Due to these discrepancies, imaging has not been advocated as the most accurate form of assessment, nor does it increase the limited understanding of tendon pathology.3,15-23 Most often, tendon pathology is diagnosed clinically based on the patient's pain narrative, previous history, and outcomes of pain provocation tests. $3,10,24,25$ Palpation may also identify pain, increased tendon thickness, and/or crepitus.3,25-29 Pain upon palpation has been found to be reliable across multiple studies when identifying tendon pathology, especially when moderate to high levels of pain upon palpation are present. ${ }^{26,27,29}$ However, when moderate to high pain levels upon palpation and patient-reported symptoms were considered together, these findings were not an adequate predictor of positive imaging findings $(p>0.05) .{ }^{26}$ These findings again reinforce the idea that symptoms and tissue pathology are not always congruent. ${ }^{3,15-23,26}$

As the uncertainty surrounding tendon pathology continues to increase across healthcare despite extensive research, alternate diagnostic

Copyright (C) by Indiana State University All rights reserved. ISSN Online 2577-8188 classification terms, such as tendinopathy and tendinalgia, have been introduced.3,30,31 Tendinopathy is an generic term to describe tendon pathology that presents with tendon pain and increased tendon thickness. ${ }^{3}$ Tendinopathy, as opposed to the previously used tendinitis or tendinosis, implies some type of tissue-based tendon pathology, without specifying a particular cause, reflecting the inconsistencies between imaging, histological, and clinical findings. 3,1 1,15-23 The newest term, tendinalgia, is an expansion of the term lateral epicondylalgia, originally coined by Waugh to describe pain at the anatomical location (i.e., lateral epicondyle) without indicating that abnormal changes in the tissue are the cause of the symptom presentation. ${ }^{30,31}$

Patient outcomes further support the notion that actual tissue changes may not be the underlying cause of a patient's symptoms. With respect to tendon pain, eccentric loading, which aims to create changes in tissue structure, is often incorporated into rehabilitation. ${ }^{3}$ However, according to a recent literature review, the use of eccentric exercise, though helpful for patient symptoms, did not result in a concomitant improvement in tendon thickness on imaging studies. 32 If tendon pathology was primarily due to a tissue abnormality, eccentric exercise should have resulted in both a change in patient symptoms and tissue structure. These discrepancies give reason to question the traditional classifications of tendon pathology, many of which imply tissue-related causes of pain (e.g., tendinitis, tendinosis). Although treatments such as eccentric exercise may be effective for reducing patient symptoms, it does not appear that these treatments are having the hypothesized effect (i.e., change in tissue structure).3,32 This may indicate that tendon pain is actually due to a pain processing dysfunction rather than actual tissue pathology. ${ }^{3,33}$ Using a more general term like tendinalgia would free the clinician to treat the source of pain as a processing dysfunction rather than a tissue pathology.

Due to the lack of consensus among imaging studies and patient outcomes, clinical assessment remains the primary strategy for evaluation and 
diagnosis. ${ }^{3,15-24}$ However, limited data exists on healthcare providers' evaluation findings. Most studies regarding tendon pathology have involved physical therapists and orthopedic surgeons, but the evaluation has often been standardized as part of a controlled study. 26,27,29 Moreover, there is little literature regarding the evaluation practices and reported findings of athletic trainers with respect to tendon pathology, even though tendon conditions are common in physically active individuals. 1,2 Despite evidence negating the validity of imaging, and therefore the emphasis on clinical evaluation, it is unknown what athletic trainers are reporting from their clinical evaluations of patients with tendon pain. By extension, it is also unknown if athletic trainers are matching their clinical diagnoses to their reported clinical findings based on the current evidence and recommendations for tendon pathology. In order to maximize the likelihood of positive treatment outcomes for patients presenting with tendon pain, it is important to gain a better understanding of the current practices of athletic trainers to identify practices that facilitate optimal treatment choices and areas needing improvement. Therefore, the purposes of this study were to assess athletic trainers': 1) documented clinical findings for patients presenting with tendon pain, 2) use of documented findings to inform clinical diagnosis of tendon pathology, and 3 ) change in tendon pathology classification when presented with a novel diagnostic term.

\section{METHODS}

This study was a retrospective descriptive analysis of a research database created and stored in Qualtrics (Provo, UT, 2002). Athletic trainers currently pursuing their doctorate in athletic training contributed to the database for this multisite research study. Clinicians were practicing in a wide range of clinical settings and working with patient populations of various physical activity levels. Participating clinicians were asked to input de-identified patient data into the database for later analysis. Before entering data, patients signed an informed consent form to allow for the inclusion of de-identified information in the

Copyright (C) by Indiana State University research database. The study protocol was approved by the University of Idaho Institutional Review Board.

\section{Procedures}

The database contained open-ended, multiple choice, and multiple select items pertaining to each portion of a standard clinical evaluation. To be included in the present study for retrospective analysis, patients had to present to the intake clinician with: 1) involvement of a specific muscle or tendon, 2) localized tendon pain, and 3) point tenderness over the involved tendon, as pain is one of the primary clinical symptoms that serve as a focus of treatment $10,14,25$ Due to the nature of the study, evaluations were not standardized. Clinicians were encouraged to perform their typical evaluation, which allowed for an authentic picture of typical athletic training practices in the evaluation of suspected tendon pathology.

Clinicians were asked to enter data regarding patient history, which included, but was not limited to: 1) age, 2) patient sport or occupation, and 3) pain scores rated on the 0-10 Numeric Pain Rating Scale (NRS; i.e., current pain, pain at best within the past 24 hours, pain at worst within the past 24 hours, pain at onset, and pain at rest). Clinicians also described pain characteristics based on the classifications set forth by Nirschl and Ashman regarding tendinosis and any objective findings (e.g., swelling, changes in tissue appearance, palpation findings, special tests performed, etc.) from the evaluation. ${ }^{14}$ It should be noted that although the classifications defined by Nirschl and Ashman are meant to describe the severity of pathology, and are therefore ordinal in nature, clinicians were allowed to choose more than one category based on patient presentation. ${ }^{14}$

At the completion of the evaluation, clinicians identified a working clinical diagnosis based on their documented findings under two separate conditions. First, they chose from a list of traditionally recognized tendon pathologies (i.e., first-, second-, or third-degree tendinitis; first or second stage tendinosis; or tendinopathy). A 
second question then asked them to classify the same tendon pain with tendinalgia, a more general term, added as a classification option to the previous list. The definition of all diagnostic terms, including tendinalgia, had been previously operationally defined and provided to the clinicians involved in the study.

\section{Data Analysis}

Data were analyzed in Statistical Package for Social Sciences (Version 25.0, IBM, Armonk, NY) and Microsoft Excel (Version 16.16.10, Microsoft, Redmond, WA). Patient cases with missing clinical diagnoses were excluded from analysis to keep sample sizes equal across analyses and to facilitate comparisons across the data. If the text entry from an open-ended response was unclear, the data was classified as "unknown". Means and standard deviations were calculated for patient age and pain scores. Frequencies and percentages were calculated for all other data to derive comparisons across documented clinical findings.

\section{RESULTS}

\section{Patient Demographics}

A total of 430 patient cases involving a primary complaint of tendon pain were extracted from the database. On average, patients were $20.7 \pm 7.3$ years old (range: 14-62 years old) and participated in over 20 different sports and/or activities. The five most common sports or activities included basketball ( $\mathrm{n}=75,17.4 \%$ ), track and field ( $\mathrm{n}=48,11.2 \%)$, soccer $(\mathrm{n}=45,10.5 \%)$, football $(n=44,10.2 \%)$, and baseball $(n=42$, $9.8 \%)$.

\section{Reported Clinical Findings}

Documented clinical findings are presented in Tables 1a-c. Pain at the site of injury was the most commonly documented clinical finding during evaluation ( $n=412,95.8 \%$ ), followed by pain that changes activity ( $n=274,63.7 \%$ ). The most commonly documented tissue changes included the presence of a tender point $(n=259,60.2 \%)$, changes in tissue tension ( $\mathrm{n}=60,14.0 \%$ ), and changes in tissue thickness ( $n=53,12.3 \%$ ), as determined from the clinician's evaluation. Overall, $87.2 \%$ of patients ( $n=375$ ) had two or fewer documented signs of inflammation (i.e., pain, loss of function, local swelling, redness, and/or heat)..$^{7-9} A$ total of 103 individuals were diagnosed with some degree (i.e., first, second, or third) of tendinitis. Of these 103 individuals, $35.0 \%(n=36)$ had pain at the site of injury as their only documented sign of inflammation. Furthermore, pain and loss of function were the only documented signs of inflammation in $10.7 \%$ ( $n=11$ ) of these 103 cases. Finally, clinicians reported using at least one orthopedic special test (e.g., tests for structural integrity of the ligaments, joint capsule, musculotendinous unit, etc.), $76.7 \%$ $(n=330)$ of the time.

Table 1a. Prevalence of Reported Inflammatory Signs

\begin{tabular}{lcc}
\hline Sign/Symptom & Frequency $(\mathbf{n})$ & Percent $(\%)$ \\
\hline Pain & 412 & 95.8 \\
Heat & 45 & 10.5 \\
Redness & 23 & 5.3 \\
Swelling & 98 & 22.8 \\
Loss of Function & 101 & 23.5
\end{tabular}

Table 1b. Prevalence of Reported Tissue Changes

\begin{tabular}{lcc}
\hline Sign/Symptom & $\begin{array}{c}\text { Frequency } \\
(\mathbf{n})\end{array}$ & $\begin{array}{c}\text { Percent } \\
(\%)\end{array}$ \\
\hline Spasm & 34 & 7.9 \\
Trigger Point & 44 & 10.2 \\
Tender Point & 259 & 60.2 \\
Change in Tissue Tension & 60 & 14.0 \\
Change in Tissue Texture & 40 & 9.3 \\
Change in Tissue Tone & 25 & 5.8 \\
Change in Tissue Thickness & 53 & 12.3 \\
Change in Sensation & 17 & 4.0 \\
\hline
\end{tabular}


Table 1c. Prevalence of Reported Pain Patterns

\begin{tabular}{lcc}
\hline \multicolumn{1}{c}{ Sign/Symptom } & $\begin{array}{c}\text { Frequency } \\
(\mathbf{n})\end{array}$ & $\begin{array}{c}\text { Percent } \\
(\%)\end{array}$ \\
\hline $\begin{array}{l}\text { Pain Post-Exercise - } \\
\text { Resolves in <24 Hours }\end{array}$ & 148 & 34.4 \\
$\begin{array}{l}\text { Pain Post-Exercise - } \\
\text { Resolves in > 24 Hours }\end{array}$ & 83 & 19.3 \\
$\begin{array}{l}\text { Pain Post-Exercise - } \\
\text { Resolves with Warm-Up }\end{array}$ & 31 & 7.2 \\
$\begin{array}{l}\text { Pain During Exercise - } \\
\text { Does Not Alter Activity }\end{array}$ & 101 & 23.5 \\
$\begin{array}{l}\text { Pain During Exercise - } \\
\text { Does Alter Activity }\end{array}$ & 274 & 63.7 \\
$\begin{array}{l}\text { Pain with Heavy ADLs } \\
\text { Pain with Light ADLs but }\end{array}$ & 126 & 29.3 \\
$\begin{array}{l}\text { Intermittent at Rest } \\
\text { Constant Pain at Rest } \\
\text { that Disturbs Sleep }\end{array}$ & 116 & 27.0 \\
\hline $\begin{array}{l}\text { Categories adaped from Nirshl } \\
\text { (1) }\end{array}$ & 38 & 8.8 \\
\hline
\end{tabular}

Categories adapted from Nirschl and

Aschman ${ }^{15}$

\section{Pain Descriptions}

Using the pain characteristics defined by Nirschl and Ashman, clinicians documented that $63.7 \%$ (n $=274$ ) of patients experienced pain that changed their activity, and patients reported that their pain after activity resolved within 24 hours in $34.4 \%(n=148)$ of cases. ${ }^{14}$ Pain scores were also recorded for each patient. The 'worst' pain ranged from $2 / 10$ to $10 / 10$ for all patients, with the average worst pain being 6.7 \pm 1.7 points. Current pain across the entire sample averaged $3.2 \pm 2.2$ points, and overall average pain (i.e., averaged current, best, and worst scores) ranged from 0.7 to 9.0, with a sample average of $4.0 \pm 1.5$ on the NRS.

Descriptive statistics for NRS scores are provided in Table 2.

\section{Classification of Tendon Pathology}

Clinician classifications of tendon pathology based on reported clinical findings are presented in Table 3 and Figure 1. Without tendinalgia as a classification option, the most

7 common clinical diagnosis was tendinopathy ( $\mathrm{n}=$ $290,67.4 \%$ ), with the second most common being some degree (i.e., first, second or third) of tendinitis ( $n=103,23.9 \%$ ). When tendinalgia was added as a classification option, there was a $46.0 \%$ decrease in the number of cases classified as tendinopathy, and tendinalgia instead became the most common diagnosis $(\mathrm{n}=$ $272,63.3 \%$ ). Of the 103 patients originally diagnosed with some degree of tendinitis, $51.5 \%$ $(n=53)$ of those diagnoses were switched to tendinalgia when this was an option.

Furthermore, of the $290(67.4 \%)$ cases originally diagnosed with tendinopathy, 125 individuals $(43.1 \%)$ were given a final diagnosis of tendinalgia.

Table 2. Pain Scores

\begin{tabular}{lcccc}
\hline Pain Score & Mean & 士SD & Minimum & Maximum \\
\hline Onset & 4.8 & 2.1 & 0.0 & 10.0 \\
Rest & 1.8 & 1.8 & 0.0 & 8.0 \\
Current & 3.2 & 2.2 & 0.0 & 10.0 \\
Best & 2.0 & 1.9 & 0.0 & 8.0 \\
Worst & 6.7 & 1.7 & 2.0 & 10.0 \\
Average $^{a}$ & 4.0 & 1.5 & 0.7 & 9.0 \\
\hline \multicolumn{4}{l}{ Average $=$ Avg(Current,Best,Worst) }
\end{tabular}

Table 3. Classification of Tendon Pathology with and without Tendinalgia as an Option

\begin{tabular}{ccc}
\hline & Without & With \\
\hline Classification & Frequency $(\mathrm{n})$ & Frequency (n) \\
Tendinitis & 103 & 38 \\
First Degree & 78 & 27 \\
Second Degree & 23 & 9 \\
Third Degree & 2 & 2 \\
Tendinosis & 37 & 28 \\
Stage I & 28 & 17 \\
Stage II & 9 & 11 \\
Tendinopathy & 290 & 92 \\
Tendinalgia & N/A & 272 \\
\hline
\end{tabular}




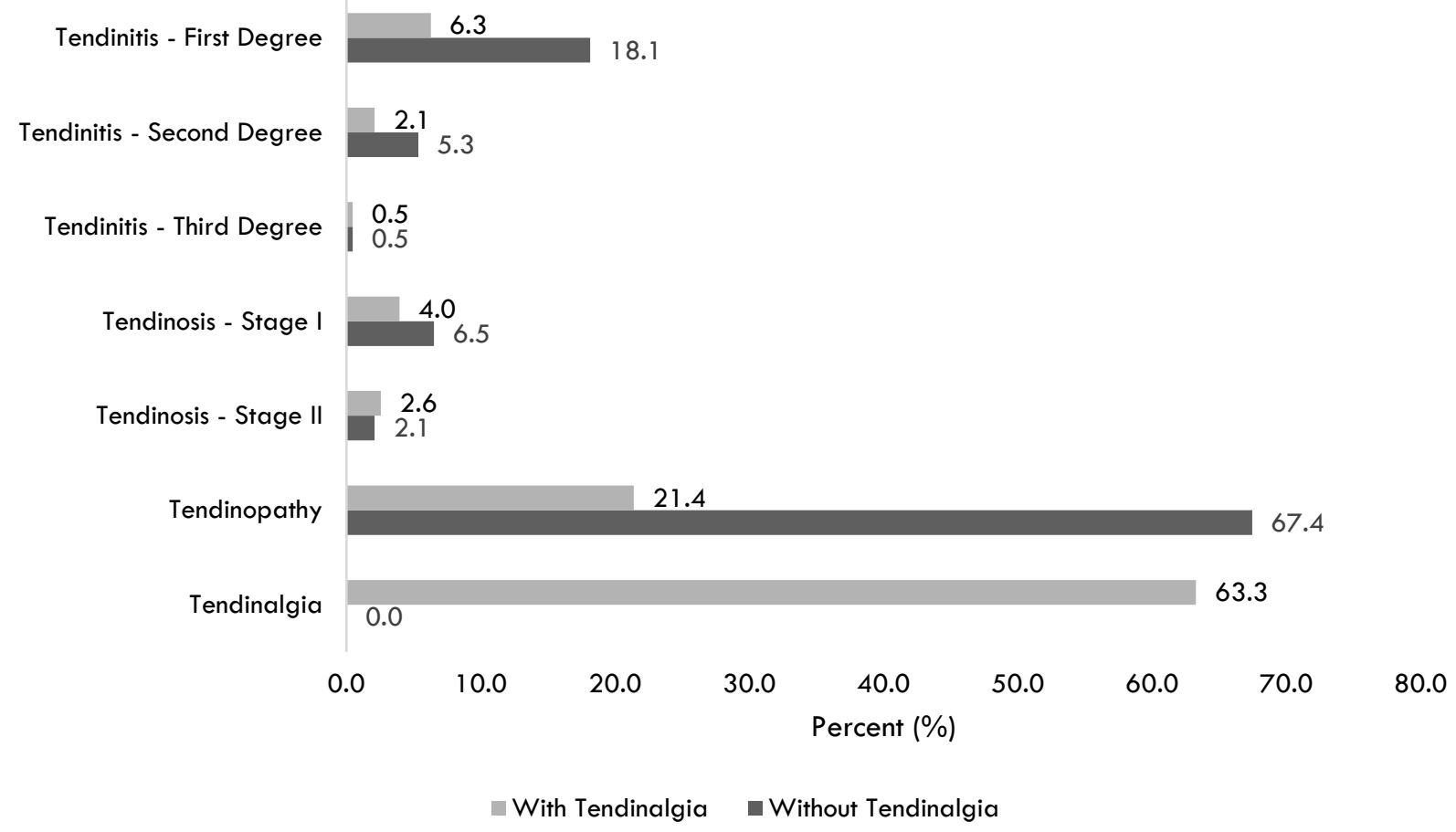

Figure 1. Classification of Tendon Pathology from Reported Clinical Findings

\section{CLINICAL APPLICATION}

Through the analysis of patient data included in a broader patient outcomes database, the frequency of various documented clinical findings in patients presenting with tendon pathology in athletic training clinics was assessed. This study also aimed to evaluate the clinical diagnoses chosen by athletic trainers based on these findings. Finally, changes in clinical diagnosis were evaluated with the introduction of the term tendinalgia, which has been proposed as an alternate term to account for the discrepancies currently surrounding tendon pathology. $3,11,15$ $23,30,31$

In the present study, the three most frequently documented clinical signs and symptoms were all related to pain (i.e., pain at site, $95.8 \%$; pain that changes activity, $63.7 \%$; tender point, $60.2 \%$ ). Theoretically, because localized pain over the involved tendon was part of the inclusion criteria for the present study, pain at the site of injury should have been reported for all patients, rather

Copyright (C) by Indiana State University All rights reserved. ISSN Online 2577-8188 than only $95.8 \%$. These inconsistencies could be due to: 1) different interpretations of the definitions between inclusion criteria and clinical findings, 2) failure to evaluate for the presence of pain at the site of injury, or 3 ) clinician error in reporting findings. Regardless of the reason, a primary finding of pain is consistent with the tendency for individuals to continue activity despite pain, only seeking treatment as the condition worsens to the point that they can no longer participate in physical activity at their desired level. $3,6,24$ The average reported pain scores from the present study (current pain: $3.2 \pm$ 2.2; worst pain: $6.7 \pm 1.7$ ) also support this pattern. Pain scores at initial evaluation (i.e., current) were high enough to allow for a decrease of at least one minimal clinically important difference on the NRS (i.e., 2 points), meaning patients would be able to identify a difference in pain after treatment, thereby giving them reason to seek care. ${ }^{34}$

Point tenderness and the general pain characteristics found in the present study were 
representative of clinical presentations outlined in the literature, but clinical diagnoses did not always align with documented signs and symptoms. $3,6,10,13,14,28$ For example, tendinitis was the second most common diagnosis when tendinalgia was not given as an option, and over one-third of patients had pain as the only documented inflammatory sign of the five cardinal signs of inflammation. However, pain by itself does not indicate a primarily inflammatory condition. Patients with tendon pathology often present with pain, but histological and imaging studies do not always support the presence of inflammation within the tissues. $3,6,10,11,14,24-27$ Therefore, pain - without other key signs or symptoms of inflammation - cannot conclusively indicate an inflammatory condition.

Loss of function was the second most documented sign of inflammation. However, because pain can affect movement, loss of function may be a notable finding regardless of the nature of the pathology (e.g., inflammatory, degenerative, etc.). ${ }^{35}$ Just over $10 \%$ of individuals diagnosed with tendinitis had pain and loss of function as their only signs of inflammation. Therefore, the accuracy of many of the tendinitis diagnoses in the present sample is questionable, though it could be argued that clinicians were aware of this possibility. Two-thirds of patients originally diagnosed with tendinitis were eventually diagnosed with tendinalgia when this was an option. It seems clinicians may not be using their documented findings to inform their clinical diagnoses when using a common, generic term like tendinitis or tendinopathy. This disconnect is a potential problem because if clinicians label a condition as inflammatory, they should also be choosing treatments that directly affect the inflammatory process, but the patient may not optimally benefit from treatment if the tendon pain is not truly inflammatory in nature. $3,11,24,25$

It is also important to ask why clinicians, who originally diagnosed their patients with tendinitis but then changed their diagnosis to tendinalgia,

Copyright (C) by Indiana State University All rights reserved. ISSN Online 2577-8188 did not originally choose tendinopathy, as tendinopathy is at least a more general term. ${ }^{3} \mathrm{In}$ identifying tendinalgia as their final diagnosis, it could be argued that clinicians demonstrated their understanding of the obscurities of tendon pathology described in the literature. $3,11,15-23,30$ Under this premise, tendinopathy would have been a more representative term given the first group of diagnostic options (i.e., without tendinalgia). ${ }^{3}$ These discrepancies could be due to: 1) clinician error in data entry, 2) failure to report clinical findings that were actually present, and/or 3) failure to consider the implications of all reported clinical findings. If clinicians are not considering the interrelatedness of all documented findings, diagnoses could be misled, again increasing the risk for ineffective treatment. ${ }^{24,25}$

Similar arguments could be made for the observed number of tendinosis diagnoses in relation to reported signs and symptoms. Increased tissue thickness was documented $12.3 \%$ of the time, but Stage I and Stage II tendinosis were only diagnosed in $8.6 \%$ of cases. Because the presence of tissue thickening was less than the number of tendinosis diagnoses, clinicians may, in this case, have considered this one sign in conjunction with the rest of their clinical findings, rather than considering it in isolation. In contrast, Nirschl and Ashman have also identified that lateral elbow tendinosis is most common in individuals in their thirties to fifties, but the majority of the patients included in the present study were younger. ${ }^{14}$ Therefore, the likelihood of true tendinosis in the present study is less probable, given the average age was just over 20 years old. Considering the average age of individuals in this study, along with the fact that imaging studies were not performed, ultimately bring into question the accuracy of the tendinosis diagnoses, and instead reinforce the use of terms tendinopathy or tendinalgia.

Despite the patterns noted previously in clinicians' classifications of tendon pathology, with $67.4 \%$ of patients being diagnosed with tendinopathy, 
diagnoses generally seemed to reflect the lack of understanding of tendon pathology described in the literature. 3,1 1,15-23,30 Moreover, when the term tendinalgia was added, there was a $46.0 \%$ decrease in the number of tendinopathy diagnoses, and a $15.0 \%$ decrease in tendinitis diagnoses. Specifically, 178 total classifications were switched from tendinitis or tendinopathy to tendinalgia. The large shifts in clinical classification to tendinalgia may reflect the lack of accurate understanding of tendon pathology. Or, it may indicate that clinicians are not effectively using their clinical findings to inform their initial working diagnosis. Under the assumption of the former, this could imply that tendinopathy may even be misleading in both describing the nature of tendon pathology and determining treatment choices. Of note, 125 of the 290 original tendinopathy cases were changed to tendinalgia when this term was included as a possible diagnosis. Perhaps the use of the term tendinalgia more accurately describes the current level of knowledge and primary findings regarding tendon pathology. Specifically, these findings suggest that tendon pain is the only consistent finding, and clinicians must perform more through examinations to determine the root cause of the tendon pain.

This exploratory study has several implications for clinical practice. There is a need for comprehensive evaluation of tendon pathology if clinicians are to more fully understand how these types of conditions manifest clinically and what causes, other than local tissue inflammation or degeneration, may be the cause of the patient's root dysfunction. According to numerous imaging studies, tissue changes can often occur without symptoms.3,15-23 For patients presenting with pain, this indicates that degenerative or inflammatory changes noted with imaging may not actually be the cause of a patient's symptoms. Moreover, if observable changes in tissue structure that appear on imaging studies do not match a patient's symptoms - or lack of symptoms - then clinical findings, which indirectly indicate the nature of

Copyright (C) by Indiana State University All rights reserved. ISSN Online 2577-8188 pathology through observation of inflammatory signs and pain provocation, may not give clinicians much further information about a patient's pain. ${ }^{3,15-25}$ This idea was supported in the present study, with many clinicians ultimately diagnosing their patients with tendinalgia, simply indicating pain at the tendon. ${ }^{30,31}$ These findings reinforce the need for detailed evaluation to determine local and regionally interdependent causes of pain and dysfunction that may result in localized tendon pain instead of treating local tissue pathology alone. $24,25,35,36$ Thus, more general terms, such as tendinalgia, may be more appropriate when classifying and labeling tendon pain.

The lack of understanding of the nature of pathology, and the possibility that clinical diagnoses do not accurately represent these pathologies, is not uncommon. There is evidence that the same is true for lateral ankle sprains, range of motion limitations, and meniscal lesions, in which tissue is the supposed cause of the dysfunction. ${ }^{36-38}$ The tissue model hypothesis has not been supported across any of these pathologies because the time to discharge, change in function, and/or resolution of pain was faster than the time that would be necessary for true tissue healing and/or changes to have occurred. ${ }^{36-39}$ While the present study did not include a treatment component, similar questions could be raised about the actual cause of tendon pain. In a case study performed by Baker et al., a patient presenting with reactive tendinopathy of the proximal biceps tendon was pain-free and discharged after three days of manual therapy treatment, with no return of symptoms after resuming physical activity. ${ }^{30}$ If the patient had reactive tendinopathy, which implies pathological tissue changes, it should have taken longer for symptoms to be eliminated, and would have most likely required intermittent rest during training to avoid the return of symptoms. ${ }^{25,39}$ Therefore, at this point in time, it does not seem appropriate for clinicians to confidently label tendon pathology based on the tissue model hypothesis. Instead, it 
seems most acceptable to describe suspected tendon pathology as pain manifesting near a tendon, which is best captured with the term tendinalgia. At the very least, clinicians should acknowledge the limitations in the clinical understanding of tendon pathology, and subsequently treat patients accordingly. Terms such as tendinalgia may help remind clinicians to treat the patient's pain as it presents, rather than treating under the assumption of an inflammatory or degenerative condition without evidence to support these notions.

\section{Limitations}

While the limited understanding of tendon pathology that exists across healthcare has been reinforced, the present study does have limitations. Several possible patterns were not assessed, including how patients may differ in clinical presentation based on sport or occupation, age, or sex, nor how clinicians may differ in their clinical diagnoses based on years of experience or other factors. Additionally, data analyzed within the database did not allow for assessment of whether or not the sign or symptom was assessed. It is possible that a sign or symptom was assessed but was not reported because it either was not present or was not documented by the clinician inputting the examination. Therefore, limitations and inaccuracies may exist within the data due to clinician error. However, the goal of the present study was to assess everyday clinical practice. Thus, completely accurate results with solely clinician judgment will be difficult to obtain no matter the nature of the study, so long as researchers and clinicians are only analyzing daily clinical practice without confirmation from imaging or other histological studies. In the future, it would be beneficial to assess: 1) patient presentation stratified by various demographic factors, 2) factors that affect clinician choice in diagnosis, and 3 ) the relationship between clinical diagnosis and treatment. Gaining a better understanding of the clinical presentation of tendon pain and clinical reasoning may

Copyright (C) by Indiana State University subsequently lead to improved treatment decisions and patient outcomes.

\section{CONCLUSION}

According to the present study, the most frequent symptom of tendon pathology appears to be the presence of pain (e.g., at the site of injury, during activity, etc.). Given the discrepancies in tendon pathology diagnosis and imaging results, clinicians must critically analyze patient presentation to identify an appropriate clinical diagnosis and subsequent treatment plan. Understanding of the term tendinalgia, like the previously introduced term of lateral epicondylalgia, may be more relevant to clinicians to improve the documentation of clinical findings, diagnostic classification, and the matching of treatments to address causes of pain. Specifically, patient outcomes may be improved if clinicians operate under the premise that tendon pathology is a pain-related condition, rather than treating from the perspective that there are inflammatory or degenerative changes within the tissue.

\section{REFERENCES}

1. Zwerver J, Bredeweg SW, van den AkkerScheek I. Prevalence of Jumper's knee among nonelite athletes from different sports: a cross-sectional survey. Am J Sports Med. 2011 ;39(9):1984-1988. https://doi.org/10.1177/0363546511413 370.

2. Scott A, Ashe MC. Common tendinopathies in the upper and lower extremities. Curr Sports Med Rep. 2006;5(5):233-241. https://doi.org/10.1007/s 1 1932-0060004-5.

3. Scott A, Docking S, Vicenzino B, et al. Sports and exercise-related tendinopathies: $A$ review of selected topical issues by participants of the second International Scientific Tendinopathy Symposium (ISTS) Vancouver 2012. Br J Sports Med. $2013 ; 47(9): 536-544$. https://doi.org/10.1136/bjsports-20130923299.

4. Andres BM, Murrell GA. Treatment of tendinopathy: what works, what does not, 
and what is on the horizon. Clin Orthop Relat Res. 2008;466(7):1539-1554. https://doi.org/10.1007/s1 1999-0080260-1.

5. Khan KM, Cook JL, Kannus P, Maffulli N, Bonar SF. Time to abandon the "tendinitis" myth. BMJ. 2002;324(7338):626-627. https://doi.org/10.1136/bmi.324.7338.62 6.

6. Khan KM, Cook JL, Taunton JE, Bonar F. Overuse tendinosis, not tendinitis. Part 1: A new paradigm for a difficult clinical problem. Phys Sportsmed. 2000;28(5):38-48. https://doi.org/10.3810/psm.2000.05.890.

7. Selvanetti A, Cipolla M, Puddu G. Overuse tendon injuries: Basic science and classification. Oper Tech Sports Med. 1997;5(3):1 10-117. https://doi.org/10.1016/\$10601872(97)80031-7.

8. Knight KL, Draper DO. Tissue response to injury: Inflammation, swelling, and edema. In Lupash E, ed. Therapeutic Modalities: The Art and Science. 2nd ed. Baltimore, MD: Lippincott Williams \& Wilkins; 2013:74-89.

9. Rocha e Silva M. A brief survey of the history of inflammation. 1978. Agents Actions. 1994;43(3-4):86-90. https://doi.org/10.1007/bf01986675.

10. Blazina ME, Kerlan RK, Jobe FW, Carter VS, Carlson GJ. Jumper's Knee. Orthop Clin North Am. 1973;4(3):665-678.

11. Cook JL, Khan KM, Harcourt PR, Grant $M$, Young DA, Bonar SF. A cross sectional study of 100 athletes with jumper's knee managed conservatively and surgically. $\mathrm{Br} J$ Sports Med. 1997;31(4):332-336. https://doi.org/10.1136/bjsm.31.4.332.

12. Puddu G, Ippolito E, Postacchini F. A classification of Achilles tendon disease. Am J Sports Med. 1976;4(4):145-150. https://doi.org/10.1177/0363546576004 00404.

13. Kaeding C, Best TM. Tendinosis: pathophysiology and nonoperative treatment. Sports Health. 2009; 1 (4):284292. https://doi.org/10.1177/1941738109337 778.

14. Nirschl RP, Ashman ES. Elbow tendinopathy: Tennis elbow. Clinics in Sports Medicine. 2003;22(4):813-836.

Copyright ( $)$ by Indiana State University All rights reserved. ISSN Online 2577-8188
https://doi.org/0.1016/S02785919(03)00051-6.

15. Boesen AP, Boesen MI, Torp-Pedersen S, et al. Associations between abnormal ultrasound color Doppler measures and tendon pain symptoms in badminton players during a season: A prospective cohort study. Am J Sports Med. 201 2;40(3):548-555. https://doi.org/10.1177/0363546511435 478.

16. Comin J, Cook JL, Malliaras P, et al. The prevalence and clinical significance of sonographic tendon abnormalities in asymptomatic ballet dancers: A 24-month longitudinal study. $\mathrm{Br} J$ Sports Med. 2013;47(2):89-92. https://doi.org/10.1136/bjsports-2012091303.

17. Docking SI, Ooi CC, Connell D. Tendinopathy: Is imaging telling us the entire story? J Orthop Sports Phys Ther. 2015;45(1 1):842-852. https://doi.org/10.2519/iospt.2015.5880.

18. Frost $P$, Andersen JH, Lundorf $E$. Is supraspinatus pathology as defined by magnetic resonance imaging associated with clinical sign of shoulder impingement? J Shoulder Elbow Surg. 1999;8(6):565-568. https://doi.org/10.1016/S10582746(99)90090-3.

19. Giombini A, Dragoni S, Di Cesare A, Di Cesare M, Del Buono A, Maffulli N. Asymptomatic Achilles, patellar, and quadriceps tendinopathy: A longitudinal clinical and ultrasonographic study in elite fencers. Scand J Med Sci Sports. 2013;23(3):311-316. https://doi.org/10.1111/i.16000838.2011.01400.x.

20. Girish G, Lobo LG, Jacobson JA, Morag Y, Miller $B$, Jamadar DA. Ultrasound of the shoulder: Asymptomatic findings in men. AJR Am J Roentgenol. 2011 ; 197(4):W713-719. https://doi.org/10.2214/AJR.11.6971.

21. Lewis J, McCreesh K, Roy JS, Ginn K. Rotator cuff tendinopathy: Navigating the diagnosismanagement conundrum. J Orthop Sports Phys Ther. 2015;45(1 1):923-937. https://doi.org/10.2519/iospt.2015.5941.

22. Paavola $M$, Kannus $P$, Paakkala $T$, Pasanen $M$, Järvinen $M$. Long-term prognosis of patients with Achilles tendinopathy: An observational 8-year follow-up study. Am J

Clinical Practice in Athletic Training Volume 3 - Issue 2 - June 2020 
Sports Med. 2000;28(5):634-642.

https://doi.org/10.1177/0363546500028 0050301.

23. Thompson SM, Fung S, Wood DG. The prevalence of proximal hamstring pathology on MRI in the asymptomatic population. Knee Surg Sports Traumatol Arthrosc. 2017;25(1):108-111. https://doi.org/10.1007/s00167-0164253-4.

24. Kaux JF, Forthomme B, Le Goff C, Crielaard JM, Croisier JL. Current opinions on tendinopathy. J Sport Sci Med. $2011 ; 10(2): 238-253$.

25. Cook JL, Purdam CR. Is tendon pathology a continuum? A pathology model to explain the clinical presentation of load-induced tendinopathy. $\mathrm{Br} J$ Sports Med. 2009;43(6):409-416. https://doi.org/10.1136/bjsm.2008.05119 3.

26. Cook JL, Khan KM, Kiss ZS, Purdam CR, Griffiths L. Reproducibility and clinical utility of tendon palpation to detect patellar tendinopathy in young basketball players. $\mathrm{Br}$ J Sports Med. 2001;35(1):65-69. https://doi.org/10.1136/bjsm.35.1.65.

27. Maffulli N, Kenward MG, Testa V, Capasso $G$, Regine R, King JB. Clinical diagnosis of Achilles tendinopathy with tendinosis. Clin J Sports Med. 2003;13(1):1 1-15. https://doi.org/10.1097/00042752$200301000-00003$.

28. Feilmeier M. Noninsertional Achilles tendinopathy pathologic background and clinical examination. Clin Podiatr Med Surg. 2017;34(2):1 29-136.

https://doi.org/10.1016/i.cpm.2016.10.00 3.

29. Hutchison AM, Evans R, Bodger O, et al. What is the best clinical test for Achilles tendinopathy? Foot Ankle Surg. 2013;19(2):112-117. https://doi.org/10.1016/i.fas.2012.12.006.

30. Baker RT, Van Riper M, Nasypany AM, Seegmiller JG. Evaluation and treatment of apparent reactive tendinopathy of the biceps brachii. Int J Athl Ther Train. 2014;19(4):14-21. https://doi.org/10.1123/ijatt.2014-0023.

31. Waugh EJ. Lateral epicondylalgia or epicondylitis: what's in a name? J Orthop

Copyright ( $)$ by Indiana State University All rights reserved. ISSN Online 2577-8188
Sports Phys Ther. 2005;35(4):200-202.

https://doi.org/10.2519/iospt.2005.0104.

32. Färnqvist K, Pearson S, Malliaras P. Adaptation of tendon structure and function in tendinopathy with exercise and its relationship to clinical outcome. 2020;29(1):107-1 15. J Sport Rehabil. https://doi.org/10.1123/isr.2018-0353.

33. Plinsinga $M L$, Brink MS, Vicenzino $B$, Wilgen $C P V$. Evidence of nervous system sensitization in commonly presenting persistent painful tendinopathies: A systematic review. J Orthop Sports Phys Ther. 2015;45(1 1):864875.

https://doi.org/10.2519/iospt.2015.5895.

34. Farrar JT, Young Jr. JP, LaMoreaux L, Werth $\mathrm{JL}$, Poole RM. Clinical importance of changes in chronic pain intensity measured on an 11 point numerical pain rating scale. Pain. 2001;94(2):149-158. https://doi.org/10.1016/S03043959(01)00349-9.

35. Cook G. Movement: Functional Movement Systems: Screening, assessment, corrective strategies. Santa Cruz, CA: On Target Publications; 2010.

36. Gamma SC, Baker RT, lorio S, Nasypany A, Seegmiller JG. A Total Motion Release warm-up improves dominant arm shoulder internal and external rotation in baseball players. Int J Sports Phys Ther. 2014;9(4):509-517.

37. Hudson R, Baker RT, May J, Reordan D, Nasypany A. Novel treatment of lateral ankle sprains using the Mulligan concept: an exploratory case series analysis. J Man Manip Ther. 2017;25(5):251-259. https://doi.org/10.1080/10669817.2017. 1332557.

38. Hudson R, Richmond A, Sanchez B, et al. An alternative approach to the treatment of meniscal pathologies: A case series analysis of the Mulligan Concept "Squeeze" technique. Int J Sports Phys Ther. 2016;11(4):564-574.

39. Lee AC, Quillen WS, Magee DJ, Zachazewski JE. Injury, inflammation, and repair: Tissue mechanics, the healing process, and their impact on the musculoskeletal system. In Magee DO, Zachazewski JE, Quillen WS, eds. Scientific Foundations and Principles of Practice in Musculoskeletal 
Point-of-Care Research: Retrospective Analysis of the Evaluation and Classification of Tendon Pathology in Athletic Training

Rehabilitation. St. Louis, MO: Saunders

Elsevier; 2007:1-22. 